\title{
An evaluation of the role of dual coding in mediating the effect of incorrectly cuing the comparison dimension in delayed matching in pigeons
}

\author{
DOUGLAS S. GRANT and SUZANNE E. MACDONALD \\ University of Alberta, Edmonton, Alberta, Canada
}

\begin{abstract}
Pigeons were trained on delayed matching-to-sample trials in which red and green sample stimuli were equally often followed by color comparisons and by line-orientation comparisons. The color samples were preceded and accompanied by cues (a triangle or a black dot) that signaled whether the comparisons on that trial would be colors or lines. Length of the retention interval was manipulated during testing, and probe trials were included on which the dimension of the comparison stimuli either was cued incorrectly or was not cued. Accuracy on incorrectly cued and on no-cue trials was less than that on correctly cued trials, and the magnitude of this effect was not influenced by the length of the retention interval. Accuracy on incorrectly cued and on no-cue trials was equivalent, and was greater than chance. The data are inconsistent with two dual-coding interpretations of the effects of incorrectly cuing the dimension of the comparison stimuli in which it is held that both retrospective and prospective sample coding occurs in this task.
\end{abstract}

The delayed matching-to-sample procedure has been employed extensively in the analysis of working memory in pigeons. In the most typical version of this task, presentation of a sample stimulus is followed by a retention interval of several seconds. Following the retention interval, two comparison stimuli are presented for a choice. The comparison stimulus that is correct on any particular trial depends upon the sample presented on that trial, and the stimulus that serves as the sample is varied from trial to trial. Because the choice response is made in the absence of the sample stimulus, accurate choice performance is believed to reflect control by information derived from the sample and retained during the retention interval.

In an interesting variation of the delayed matching procedure, Stonebraker and Rilling (1984) trained pigeons in a task in which color samples (red and green fields) were equally often followed by color comparisons and by line-orientation comparisons. Superimposed on the sample, and extending throughout the retention interval, was a cue (either a circle or a triangle) that signaled whether the comparison stimuli on that trial would be colored fields or line orientations. The circle signaled that color comparisons would be presented, and the triangle signaled that line-orientation comparisons would be presented. Follow-

This research was supported by Grant A0443 from the Natural Sciences and Engineering Research Council of Canada (NSERC) awarded to the first author. The second author was supported by a postgraduate scholarship awarded by NSERC. Suzanne MacDonald is now at the Department of Psychology, University of British Columbia, Vancouver, British Columbia V6T 1Y7, Canada. Correspondence concerning this article should be addressed to Douglas S. Grant, Department of Psychology, University of Alberta, Edmonton, Alberta T6G 2E9, Canada. ing training, probe trials were introduced in which the comparison dimension was cued incorrectly. Specifically, a triangle was presented on probe trials that terminated in color comparisons, and a circle was presented on probe trials that terminated in line-orientation comparisons.

Stonebraker and Rilling (1984) found that accuracy was 17.3 percentage points lower on incorrectly cued trials (72.2\% correct) than on correctly cued trials $(89.5 \%$ correct). In a recent replication and extension of Stonebraker and Rilling's research, Santi, Musgrave, and Bradford (1988) conducted four tests in which accuracy on correctly and incorrectly cued trials was compared. Accuracy was lower on incorrectly cued trials than on correctly cued trials in all four tests, and the magnitude of the difference in accuracy ranged from 2.9 to 12.5 percentage points. Mean percentage of correct responses on incorrectly cued trials ranged from 78.0 to 87.5.

Both Stonebraker and Rilling (1984) and Santi et al. (1988) interpreted the finding that accuracy is reduced by incorrectly cuing the comparison dimension as implicating processes of prospective coding. Specifically, they argued that the cues exerted control over which comparison response instruction was activated by the color sample. To illustrate, consider a trial on which a red sample is presented. If the comparison dimension cue on that trial was associated with color comparisons, then the red sample would tend to activate a response instruction to peck a particular color comparison (e.g., peck the red comparison). If, on the other hand, the comparison dimension cue on that trial was associated with line comparisons, then the red sample would tend to activate a response instruction to peck a particular line comparison (e.g., peck the vertical comparison). 
It should be noted, however, that the reduction in accuracy on incorrectly cued trials is not sufficient to justify the stronger conclusion that sample coding in this procedure is exclusively prospective. In particular, both prospective sample coding, in which the pigeon encodes information about the comparison stimuli, and retrospective sample coding, in which the pigeon encodes information about the sample stimuli, may occur in the cuing task. The possibility that pigeons employ both prospective and retrospective coding concurrently in delayed matching tasks has been noted by numerous investigators (e.g., Honig \& Thompson, 1982; Santi et al., 1988; Urcuioli \& Zentall, 1986; Wasserman, 1986), and such a dual-coding perspective provides a ready account of Stonebraker and Rilling's (1984) and Santi et al.'s (1988) findings.

According to a dual-coding view, reduced accuracy on incorrectly cued trials is mediated by processes of prospective coding, and above-chance accuracy on incorrectly cued trials is mediated by processes of retrospective coding. The present experiments were designed to test two different interpretations, both derived from a dual-coding perspective, of the effect of incorrectly cuing the comparison dimension. According to one interpretation, evaluated in Experiment 1, the sample stimulus activates (and/or establishes) both a prospective code and a retrospective code on each trial. According to a second interpretation, evaluated in Experiment 2, the sample activates (and/or establishes) either a prospective code or a retrospective code on each trial.

\section{EXPERIMENT 1}

One could account for the effect of incorrectly cuing the comparison dimension by assuming that the sample stimulus is encoded both prospectively and retrospectively on each trial. In order for a dual-coding model to account for the reduction in accuracy produced by miscuing the comparison dimension, it is necessary to assume that the retrospective code is less durable than the prospective code (either because of properties inherent in the two types of codes or because only the prospective code is rehearsed during the retention interval). In this view, accuracy on incorrectly cued trials should be less than that on correctly cued trials, but should exceed $50 \%$ correct. This is the case because (1) performance on correctly cued trials will typically be controlled by a prospective code, (2) performance on incorrectly cued trials can only be controlled by a retrospective code, and (3) the retrospective code is lost during the retention interval on the majority of trials. These notions appear to form the basis of Maki's suggestion (cited in Stonebraker \& Rilling, 1984) that the use of a short retention interval in the miscuing procedure might be diagnostic of the form of coding employed. Moreover, Santi et al. (1988, pp. 97-98) offered an interpretation of the cuing phenomenon similar to the dual-coding account under present consideration.
Although the dual-coding account provides a plausible interpretation of the effect of miscuing the comparison dimension, the account has not been tested directly. The present experiment was designed to provide such a test and involved the manipulation of retention-interval length. In contrast to prior research in which each bird was tested at only a single retention interval (Santi et al., 1988; Stonebraker \& Rilling, 1984), the birds in the present experiment were tested on correctly and incorrectly cued trials in which the retention test was equally often immediate $(0-\mathrm{sec}$ delay) and delayed $(M=6.6 \mathrm{sec}$, range 3-9 sec). The dual-coding account predicts that incorrectly cuing the comparison dimension will reduce accuracy less strongly on the immediate test because the retrospective code is more likely to be available to guide accurate performance. An even stronger prediction follows from Farthing, Wagner, Gilmour, and Waxman's (1977) suggestion that choice behavior on an immediate test is controlled exclusively by a retrospective code. In this view, accuracy on the immediate test should be unaffected by whether the dimension of the comparison stimuli is cued correctly or incorrectly.

The method of cue presentation employed in the present experiment differed from that employed by Stonebraker and Rilling (1984) and Santi et al. (1988) in two aspects. First, to avoid confounding length of the retention interval and length of exposure to the comparison dimension cue, the comparison dimension cue was not presented during the retention interval. This contrasts with prior research in which the comparison dimension cue was presented either throughout the retention interval (Santi et al.'s "simultaneous" procedure and Stonebraker \& Rilling's procedure) or during the first $1 \mathrm{sec}$ of the retention interval (Santi et al.'s "successive" procedure). Second, in the present experiment, the comparison dimension cue both preceded and accompanied the sample stimulus. In contrast to prior research (Santi et al.'s "simultaneous"' procedure and Stonebraker \& Rilling's procedure), the comparison dimension cue was not superimposed on the sample, but was instead presented on the side keys during sample presentation (the sample stimulus was presented on the center key). It was anticipated that having the cue accompany the sample, but remain physically distinct from the sample, would be particularly conducive to the cues acquiring a supraordinal role in which they determined the specific response instruction activated by the color sample.

\footnotetext{
Method

Subjects. Seven experimentally naive Silver King pigeons were reduced to and maintained at $80 \%$ of their free-feeding weights.

Apparatus. The birds were tested in seven identical chambers. Three pecking keys were mounted horizontally in a row $20 \mathrm{~cm}$ above the floor in each chamber. An Industrial Electronics, Inc. in-line projector was mounted behind each key and was used to project stimuli onto the pecking key. A grain feeder was mounted below the center pecking key. Each test chamber was enclosed in a soundand light-attenuating enclosure. Masking noise was provided by an
} 
exhaust fan within the enclosure and by white noise delivered through a speaker in the testing room. The presentation of events within the chambers and the recording of data were accomplished using microcomputers.

Procedure. The birds were trained to eat grain from the magazine and were then autoshaped to peck red and green fields and horizontal and vertical line orientations. After each bird was responding to each of the stimuli reliably, training on 0 -sec delayed matching-to-sample began. Each trial began with the presentation of either a white triangle on a black background or a black dot on a white background on the center key. The first response to occur after $2 \mathrm{sec}$ had elapsed caused all three keys to be illuminated. The sample stimulus, either red or green, was presented on the center key, and the same stimulus that had appeared on the center key prior to sample presentation was now presented on each of the side keys. Following a 5-sec exposure, the center key was darkened and comparison stimuli replaced the triangles or circles on the side keys. On trials in which the triangle preceded and accompanied the sample, the comparison stimuli consisted of one red field and one green field. A response to the comparison that matched the sample was designated correct. On trials in which the dot preceded and accompanied the sample, the comparison stimuli consisted of one horizontal line orientation and one vertical line orientation. A response to the vertical line was designated correct if the sample had been red, and a response to the horizontal line was designated correct if the sample had been green. In all cases, a single response to either comparison caused all three keys to be darkened. Correct responses resulted in a 3-sec access to grain, and incorrect responses resulted in a 3-sec timeout. An intertrial interval of $20 \mathrm{sec}$ preceded each trial.

Each session of 0-sec delayed matching consisted of 80 trials, 40 involving color comparisons (and hence the triangle cue) and 40 involving line comparisons (and hence the dot cue). The sample was equally often red and green on both color tests and line tests. The left-right position of the correct comparison stimulus was balanced within sample type and comparison dimension. Training on 0-sec delayed matching continued for 64 sessions.

Following 0 -sec delayed matching training, sessions were altered such that half the trials involved a delay greater than $0 \mathrm{sec}$. The delay on these trials was initially set at $1 \mathrm{sec}$. The delay interval was incremented gradually on a subject-by-subject basis until each bird reached a delay at which accuracy stabilized at approximately $75 \%$ correct. One bird reached a terminal delay of $3 \mathrm{sec}, 2$ reached a terminal delay of $5 \mathrm{sec}, 1$ reached a terminal delay of $6 \mathrm{sec}$, and 3 reached a terminal delay of $9 \mathrm{sec}$. Sample type, comparison dimension, and position of the correct comparison stimulus were balanced within delay. Each bird received 120 sessions of training in this phase, and each received a minimum of 20 sessions at the terminal delay value.

Experimental testing followed delayed matching training and involved two types of sessions-baseline sessions and probe sessions. Baseline sessions were identical to those of training in which the terminal delay value was employed. Probe sessions were identical to baseline sessions, except that $\mathbf{1 6}$ of the $\mathbf{8 0}$ trials were incorrectly cued trials (the remaining 64 trials were correctly cued and were identical to those of training). On incorrectly cued trials, the dot preceded and accompanied the sample on color tests, and the triangle preceded and accompanied the sample on line tests. The relation between sample color and correct comparison stimulus was the same as on correctly cued trials, and correct responses were reinforced. Each of the 16 types of incorrectly cued trials ( 2 sample types $\times 2$ comparison dimensions $\times 2$ delay intervals $\times 2$ positions of the correct comparison) was tested once in each probe session. Each of the 16 types of correctly cued trials was tested four times in each probe session. Only data from probe sessions were recorded. Experimental testing involved two phases of test-

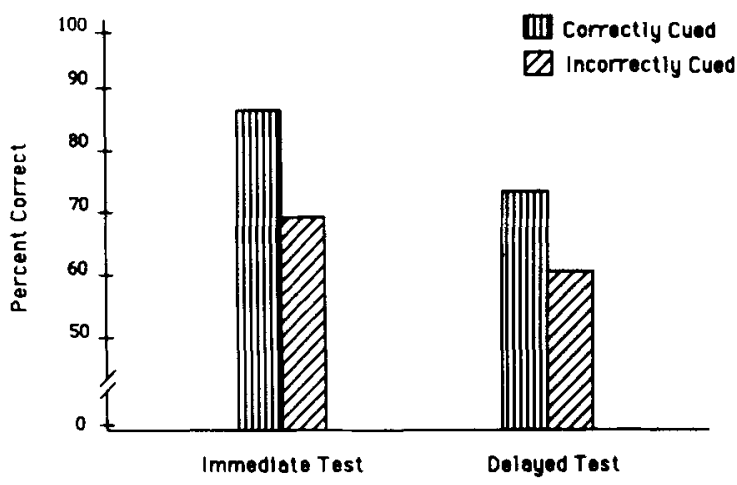

Figure 1. Percentage of correct responses on correctly cued and incorrectly cued trials on the immediate (0-sec delay) and delayed (mean delay $=6.6 \mathrm{sec}$, range 3-9 sec) tests in Experiment 1 .

ing separated by 20 baseline sessions. Each testing phase involved eight probe sessions and each probe session was preceded by two baseline sessions.

\section{Results}

Accuracy on correctly cued and incorrectly cued trials on the immediate and delayed tests is shown in Figure 1. Collapsed across the delay factor, accuracy was considerably higher on correctly cued trials $(80.3 \%$ correct) than on incorrectly cued trials $(65.0 \%$ correct). Moreover, the magnitude of this discrepancy was not affected strongly by length of the retention interval. Accuracy was 17.3 percentage points lower on incorrectly cued trials than on correctly cued trials on the immediate test, and was 13.3 percentage points lower on the delayed test. A trial type (correctly cued and incorrectly cued) $\times$ delay (immediate test and delayed test) $\times$ test blocks ( 1 and 2 ) analysis of variance revealed significant main effects of trial type $[F(1,6)=35.99, p<.01]$ and delay $[F(1,6)=$ $15.03, p<.01]$. The main effect of test blocks was not significant $(p>.05)$, and none of the interaction terms was significant.

\section{Discussion}

The present findings are consistent with those of others (Santi et al., 1988; Stonebraker \& Rilling, 1984) in that (1) accuracy on incorrectly cued trials was reduced relative to that on correctly cued trials, and (2) accuracy on incorrectly cued trials exceeded chance ( $50 \%$ correct). The novel finding of the present experiment was that the discrepancy between accuracy levels on correctly and incorrectly cued trials was equivalent on immediate and delayed tests. The latter finding is inconsistent with the notion that above-chance accuracy on incorrectly cued trials is mediated by a retrospective code, which, although less durable than a prospective code, may occasionally be available to direct performance. According to that hypothesis, the effects of incorrectly cuing the dimension of the comparison stimuli should be greater on a delayed test than on an immediate test. 


\section{EXPERIMENT 2}

The first experiment tested and failed to support a dualcoding interpretation of the effects of incorrectly cuing the comparison dimension. It had been maintained that (1) presentation of a sample stimulus activates (and/or establishes) both a retrospective and a prospective code, and (2) prospective codes are more resistant to forgetting than are retrospective codes. In the present experiment, an alternative dual-coding interpretation was evaluated. This interpretation assumes that a sample is coded either prospectively or retrospectively on any particular trial. To account for the reduction in accuracy produced by miscuing the comparison dimension, it is necessary to assume that the availability of comparison dimension cues increases the probability that a sample will be coded prospectively rather than retrospectively. The main difference between the present interpretation and the interpretation considered in Experiment 1 is that the present version assumes that only one code is active on each trial, rather than that two codes, differing in terms of durability, are active on each trial. Therefore, the finding that the magnitude of the miscuing effect is not influenced by the length of the retention interval is not problematic for the present interpretation.

The dual-coding interpretation under present consideration leads to the expectation that matching accuracy should be higher on no-cue trials than on incorrectly cued trials. This prediction follows from the notion that the probability of prospective coding should be higher on incorrectly cued trials, in which comparison dimension cues accompany the sample, than on no-cue trials, in which comparison dimension cues are not presented. It is also the case, therefore, that the probability of retrospective sample coding should be higher on no-cue trials than on incorrectly cued trials. If a retrospective code is responsible for accurate performance in the absence of an appropriate prospective code, then accuracy should be higher on no-cue trials than on incorrectly cued trials. To test this prediction, on some probe trials, no comparison dimension cue was presented during experimental testing. On these no-cue trials, the red or green sample was neither preceded nor accompanied by the comparison dimension cues. Except for the inclusion of no-cue probe trials, the design of the present experiment was identical to that of the first experiment.

Santi et al. (1988) also compared accuracy on incorrectly cued and no-cue trials, and in three of four cases accuracy on no-cue and incorrectly cued trials was equivalent. In one case, however, accuracy on no-cue trials (87.1\% correct) was significantly higher than that on incorrectly cued trials $(80.5 \%$ correct). The latter finding is difficult to interpret not only because in the majority of cases no difference between accuracy on incorrectly cued and no-cue trials was found, but also because Santi et al.'s birds had received extensive preexperimental training in standard (i.e., noncued) delayed matching involving color and/or line stimuli. Therefore, performance on no-cue trials could have been mediated, in whole or in part, by coding processes acquired during the earlier training. That is, the birds previously exposed to color matching must have learned to code the color samples either prospectively or retrospectively, and either coding process could mediate accurate performance on no-cue trials. The birds tested in the present experiment had not experienced no-cue trials until such trials were introduced during experimental testing. In the present case, therefore, performance on no-cue trials must necessarily reflect coding processes acquired during the cuing procedure, and the present data are therefore relevant to the question of whether above-chance accuracy on incorrectly cued trials might reflect a retrospective code that is available on the minority of incorrectly cued trials.

\section{Method}

Subjects and Apparatus. The subjects and apparatus were the same as in Experiment 1.

Procedure. Immediately following the completion of testing in Experiment 1, each bird received 20 consecutive sessions of baseline training consisting of 80 correctly cued trials. The baseline sessions were identical to those of Experiment 1. Experimental testing immediately followed the 20 baseline sessions.

Experimental testing involved a total of 24 sessions -16 baseline sessions and 8 probe sessions. Baseline sessions involved 80 correctly cued trials and were identical to the baseline sessions in Experiment 1. Probe sessions consisted of 48 correctly cued trials, 16 incorrectly cued trials, and 16 no-cue trials. Correctly cued and incorrectly cued trials were identical to those of Experiment 1 . Nocue trials were identical to correctly cued and incorrectly cued trials, with the exception that the comparison dimension cue was omitted. That is, the color sample was neither preceded nor accompanied by any additional stimuli. The relation between sample color and correct comparison stimulus was the same as that on correctly and incorrectly cued trials, and correct responses were reinforced. Each of the 16 types of no-cue trials $(2$ samples $\times 2$ comparison dimensions $\times 2$ delay intervals $\times 2$ positions of the correct comparison) was tested once in each probe session. Each probe session was preceded by two baseline sessions, and only data from probe sessions were recorded. All other aspects of the procedure were the same as in Experiment 1.

\section{Results}

Accuracy on correctly cued, incorrectly cued, and nocue trials on the immediate and delayed tests is shown in Figure 2. A trial type (correctly cued, incorrectly cued,

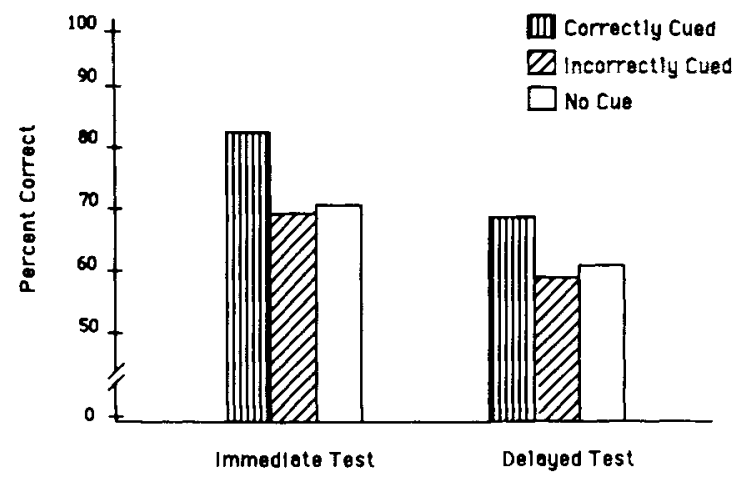

Figure 2. Percentage of correct responses on correctly cued, incorrectly cued, and no-cue trials on the immediate (0-sec delay) and delayed (mean delay $=6.6 \mathrm{sec}$, range 3-9 sec) tests in Experiment 2 . 
and no cue) $\times$ delay (immediate and delayed tests) analysis of variance revealed significant main effects of trial type $[F(2,12)=11.06, p<.01]$ and delay $[F(1,6)=$ $30.57, p<.01]$. The interaction was not significant $[F(2,12)=1.22]$. Newman-Keuls tests $(p=.05)$ revealed that accuracy on correctly cued trials $(75.9 \%$ correct) was significantly different from that on both incorrectly cued trials $(64.7 \%$ correct) and no-cue trials $(66.1 \%$ correct), and accuracy on incorrectly cued and no-cue trials did not differ.

\section{Discussion}

The present experiment replicated the three main findings from Experiment 1: (1) accuracy on incorrectly cued trials was lower than on correctly cued trials, (2) accuracy on incorrectly cued trials exceeded chance, and (3) the magnitude of the accuracy discrepancy on correctly and incorrectly cued trials was not greater on a delayed test than on an immediate test. In addition, the present experiment revealed that accuracy on no-cue trials was not significantly higher than that on incorrectly cued trials. The latter finding fails to support a dual-coding interpretation in which it is held that above-chance accuracy on incorrectly cued trials is mediated by a retrospective code that is available on the minority of incorrectly cued trials. As discussed earlier, such an account anticipates that retrospective coding should occur more frequently on nocue trials than on incorrectly cued trials. If that were so, then accuracy on no-cue trials should have been significantly higher than that on incorrectly cued trials.

\section{GENERAL DISCUSSION}

The present results replicate Stonebraker and Rilling's (1984) and Santi et al.'s (1988) findings that accuracy on trials in which the comparison dimension is cued incorrectly (1) is reduced relative to that on trials in which the dimension of the comparison stimuli is cued correctly, and (2) is greater than chance (50\% correct). The present research explored the possibility that an adequate account of these effects might be provided by a dual-coding perspective in which it is held that processes of both prospective and retrospective coding are involved in the cuing procedure. According to this perspective, the finding that accuracy on incorrectly cued trials is reduced relative to that on correctly cued trials is mediated by processes of prospective coding, whereas the finding that accuracy on incorrectly cued trials is above chance is mediated by processes of retrospective coding.

Two interpretations of the effects of miscuing the comparison dimension, each derived from the dual-coding perspective, were evaluated experimentally. According to one account, both a prospective code and a retrospective code are activated (and/or established) on each trial, and the prospective code is more likely than the retrospective code to persist throughout the retention interval. The finding that accuracy on an immediate test was reduced as markedly by miscuing as was that on a delayed test failed to support this account. According to the second account, either a prospective code or a retrospective code is activated (and/or established) on each trial, and the availability of comparison dimension cues causes the animal to code prospectively on the majority of trials. The finding that accuracy on no-cue trials was not significantly higher than that on incorrectly cued trials failed to support the second account. In our view, the two interpretations evaluated in the present experiments are exhaustive of those that are derivable from a dual-coding perspective. The present findings therefore permit rejection of the notion that the effects of miscuing the comparison dimension reflect the operation of both prospective and retrospective coding.

The present experiments were designed to evaluate empirically two interpretations of the miscuing effect derivable from a dual-coding viewpoint, and were not designed to identify the most viable account of the miscuing effect. Nonetheless, it is worthwhile to note that, in our view, a purely prospective account, originally offered by Stonebraker and Rilling (1984), does provide an adequate account of the effect of incorrectly cuing the dimension of the comparison stimuli.

As noted earlier, the view that the cues influence which prospective code is activated by the color sample correctly anticipates that accuracy will be reduced by cuing the comparison dimension incorrectly. However, both Honig and Thompson (1982, p. 243) and Santi et al. (1988, p. 88) have argued that the fact that accuracy is well above chance on incorrectly cued trials is difficult to interpret within a prospective model. Moreover, Santi et al. have also argued that a prospective view anticipates higher accuracy on no-cue trials than on incorrectly cued trials, an effect that was not obtained in our second experiment. In our view, however, neither of these findings requires abandonment of a purely prospective interpretation.

Consider first the finding that accuracy on incorrectly cued trials is well above chance. There are several ways in which a prospective model could accommodate this finding. For example, it could be assumed that although presentation of a comparison dimension cue increases the probability that the color sample will activate the code appropriate to that dimension, on some proportion of the trials, the color sample activates the code appropriate to the noncued dimension. If this is so, accuracy on incorrectly cued trials should be lower than that on correctly cued trials, but should exceed chance. Thus, the fact that accuracy on incorrectly cued trials is above chance does not challenge a prospective account.

Consider next the finding that accuracy on no-cue trials is equivalent to that on incorrectly cued trials. In our view, this finding is not relevant to an evaluation of the adequacy of a single-process prospective account. This is the case because the prospective view does not permit unambiguous specification of the result of failing to present a cue. It is clear that, according to a prospective view, the probability that a sample will activate an irrelevant comparison code should be higher on incorrectly cued trials 
than on no-cue trials. This result would tend to produce higher accuracy on no-cue trials than on incorrectly cued trials. However, this tendency could be mitigated or even overridden by an increased probability that a noncued sample will fail to activate either prospective code. The latter process would tend to depress accuracy toward a chance level on no-cue trials. A prospective account can therefore accommodate any relation between accuracy on no-cue and incorrectly cued trials, thus rendering such a comparison irrelevant to an assessment of the adequacy of a single-process prospective account.

The above discussion should not be taken to imply that a prospective interpretation is the only viable account of cuing effects. Indeed, at least three alternative interpretations, two of which exclusively employ processes of retrospective coding, may be offered. One account, suggested by a reviewer of an earlier version of the present report, maintains that pigeons retrospectively code the color samples and also develop an expectation that the comparisons will be from a particular dimension. In this view, accuracy is reduced on miscued trials because the presentation of the unexpected comparison dimension disrupts accuracy. On no-cue trials, accuracy is reduced by generalization decrement arising because the sample presentation on such trials is novel in that the color sample is not accompanied by a comparison dimension cue.

The two remaining accounts each maintain that the nominal sample and the nominal cue are actually processed as a compound sample; the accounts differ in terms of whether the compound is held to be coded retrospectively or prospectively. D'Amato (cited in Honig \& Thompson, 1982), commenting on an unpublished report of Stonebraker and Rilling's (1984) findings, noted that the effects of miscuing the comparison dimension could be accounted for by assuming that (1) the superimposed color sample and form cue is processed as a compound sample, (2) the compound sample is coded retrospectively, and (3) processes of stimulus generalization operate at the time of testing such that the retrospective code may evoke responding to a comparison stimulus with which it is not directly associated. Although the comparison dimension cue was not superimposed on the sample either in Santi et al.'s (1988) successive procedure or in the procedure employed in the present experiments, it is nonetheless possible that the cue and the sample were coded retrospectively as a compound. An alternative account, which also maintains that the nominal sample and the nominal cue are processed as a compound, may be offered by extending Grant and MacDonald's (1986) prospective coding interpretation of matching to element and compound samples to the cuing task. Such an account is similar to that of D'Amato, except that (1) the samplecue compounds are held to be encoded prospectively rather than retrospectively, and (2) processes of stimulus generalization are held to operate at the time of instructional code activation rather than at the time of testing.
In our view, the comparison dimension cuing task does not afford a means to discriminate between the various accounts considered above in which a single coding process is employed. It should be noted in particular that the indeterminate nature of models employing a single coding process precludes the derivation of precise, noncontentious predictions concerning the anticipated effects on accuracy of (1) miscuing and (2) failing to present a cue. Until such models are formulated more rigorously, one can do no more than attempt to evaluate the relative plausibility of the interpretations of the effects of miscuing and failing to present a comparison dimension cue afforded by these models.

The view that the comparison dimension cuing task is unlikely to play a prominent role in discriminating between models employing a single coding process does not, however, preclude the possibility of employing the cuing task as an analytical tool to assess particular theoretical propositions concerning coding processes. The present experiments did, of course, employ the latter tactic. The basic notion was that if dual coding is involved in the cuing task, it should be revealed by tests in which the sample was sometimes not accompanied by a cue and by manipulations of delay-interval length. The fact that no evidence of dual coding was obtained calls into question the involvement of dual-coding processes not only in the cuing task but in the delayed matching task in general. In our view, pigeons should be no less likely to employ dual coding in the cuing task than in the standard delayed matching task and, therefore, the present results militate against the notion that processes of dual coding are typically involved in delayed matching tasks.

\section{REFERENCES}

Farthing, G. W., Wagner, J. M., Gilmour, S., \& Waxman, H. M (1977). Short-term memory and information processing in pigeons. Learning \& Motivation, 8, 520-532.

Grant, D. S., \& MacDonald, S. E. (1986). Matching to element and compound samples in pigeons: The role of sample coding. Journal of Experimental Psychology: Animal Behavior Processes, 12, 160-171.

HoniG, W. K., \& Thompson, R. F. K. (1982). Retrospective and prospective processing in animal working memory. In G. H. Bower (Ed.), The psychology of learning and motivation: Advances in research and theory (Vol. 16, pp. 239-283). New York: Academic Press.

Santi, A., Musgrave, S., \& Bradford, S. A. (1988). Utilization of cues signaling different test stimulus dimensions in delayed matching to sample by pigeons. Learning \& Motivation, 19, 87-98.

Stonebraker, T. B., \& Rilling, M. (1984). Retrospective versus prospective processes in delayed matching to sample. Bulletin of the Psychonomic Society, 22, 372-375.

Urculoli, P. J., \& Zentall, T. R. (1986). Retrospective coding in pigeons' delayed matching-to-sample. Journal of Experimental Psychology: Animal Behavior Processes, 12, 69-77.

Wasserman, E. A. (1986). Prospection and retrospection as processes of animal short-term memory. In D. F. Kendrick, M. E. Rilling, \& R. R. Denny (Eds.), Theories of animal memory (pp. 53-75). Hillsdale, NJ: Erlbaum.

(Manuscript received August 14, 1989; revision accepted for publication November 30, 1989.) 